\title{
Stellar Rotation in Galactic Clusters
}

\author{
Douglas R. Gies and Wenjin Huang \\ Center for High Angular Resolution Astronomy, Department of Physics \\ and Astronomy, Georgia State University, Atlanta, GA 30303, U.S.A.
}

\begin{abstract}
Open clusters offer us the means to study stellar properties in samples with well-defined ages and initial chemical composition. Measurements of the projected rotational velocities for large numbers of cluster stars are becoming practical with the advent of multi-object spectroscopy, and such data make it possible to study the time evolution of the rotational distributions of massive stars. Investigations of these rotational distributions provide information on the angular momentum distribution in newborn clusters, the rotational spin-down due to wind loss, magnetic fields, and binary companions, and the incidence of spin-up due to mass transfer in close binaries. Here we review some of the recent observational studies and highlight some preliminary results from our work.
\end{abstract}

\section{Introduction}

At the first meeting on Stellar Rotation, Abt (1970) summarized the pioneering work on the rotational velocity characteristics of stars in open clusters. There were investigations available then on the projected rotational velocities $(v \sin i)$ of stars in 15 clusters and associations, and Abt showed that there are real differences among the $v \sin i$ distributions due to evolutionary expansion, tidal effects in close binaries, and magnetic breaking. All these processes cause a spin down among members of the massive star population. Since that time we have witnessed a huge growth in the number of measurements of projected rotational velocity among cluster stars (particularly among the lower mass population; Mathieu 2004; Stauffer 2004), a trend which is now accelerating. A fairly complete census of the results to date comes from the WEBDA open cluster database ${ }^{1}$ maintained by J.-C. Mermilliod (Mermilliod 1995). The WEBDA database lists 175 papers on $v \sin i$ that provide $v \sin i$ data for 3151 stars in 107 clusters. However, the coverage is sparse in many cases, and there are only 16 clusters for which measurements exist for at least 50 stars. The situation for the rare massive stars is less satisfactory since there are only 5 clusters with measurements for at least 50 stars of spectral types O, B, and A (cf. Levato et al. 1991; Morrell \& Levato 1991). This review focuses on the rotational characteristics of the massive star population in clusters with a particular emphasis on stars in the evolutionary stage between the zero age main sequence (ZAMS)

\footnotetext{
${ }^{1}$ http: //obsww . unige.ch/webda/
} 
and the terminal age main sequence (TAMS) (see Stauffer 2004 for a discussion about the low mass stars in clusters). We explore the processes that affect stellar rotation in these stars and we describe preliminary results from a survey we have made of massive stars in young galactic clusters.

\section{Rotational Evolution of Massive Stars}

The spin evolution of a single, non-magnetic, massive star during its main sequence lifetime is driven by the angular momentum loss in the stellar wind, a net increase in the moment of inertia, and an increase in stellar radius. Realistic models of these processes were recently presented independently by Heger \& Langer (2000) and Meynet \& Maeder (2000). These models describe both evolutionary changes in the stellar interior and the resulting changes in equatorial rotational velocity and photospheric chemical abundances. The models generally predict that massive stars spin down during core $\mathrm{H}$-burning at a rate that is larger in more massive stars and in stars with higher initial rotational speeds.

An exception to the spin down trend can occur very close to the TAMS where the models predict a brief episode of increased rotational speed as the core contracts prior to $\mathrm{H}$-shell burning. In some cases, this increase may lead to near critical rotational velocities and the kind of enhanced equatorial mass outflows that are observed in rapidly rotating Be stars (Maeder et al. 1999; Zorec 2004). There is observational evidence that the incidence of Be stars tends to peak in open clusters in the age interval 13 - 25 Myr (Fabregat \& Torrejón 2000), approximately when early B-type stars are nearing the TAMS. However, Be stars are not found exclusively near the TAMS in the Hertzsprung-Russell diagram (HRD) but appear to cover the entire range between ZAMS and TAMS (Mermilliod 1982; Zorec 2004).

Massive stars in close binary systems have lower $v \sin i$ values than those found in single stars. A seminal investigation of rotation among stars in the Sco OB2 association by Brown \& Verschueren (1997) demonstrated that members of the binary population rotate more slowly than the single stars of the association (see their Fig. 9). Tidal forces in close binaries can readily act to slow rotation to synchronous rates (Rieutord 2004), but these forces are probably most effective in very close binaries. For example, Abt, Levato, \& Grosso (2002) show that binaries containing B-type stars are synchronously rotating for orbital periods less than 2.4 days. Most of the detected binary systems in surveys like those of Brown \& Verschueren have orbital periods longer than this limit (sometimes much longer), so that tidal friction is probably insufficient to explain the general slow rotation of binaries. The star formation process may favor allocating the angular momentum of the natal cloud into orbital motion instead of rotation in the case of binaries.

Magnetic spin down is also present in a subset of massive stars with evidence of magnetic fields (primarily among the helium-peculiar B stars and Bp - Ap stars; Neiner 2002; Mathys 2004). The combined factors lead to an inverse correlation between the incidence of binaries plus magnetic stars in open clusters and the cluster mean $v \sin i$ relative to the field star population (Abt \& Sanders 1973; Morrell \& Levato 1991; Abt \& Willmarth 1999; Abt et al. 2002). 
All of the processes discussed thus far cause a spin down in massive stars. The remaining evolutionary process we need to consider is mass transfer in close binary stars, which may cause a spin up of the mass gainer. Many early type stars are found in close binaries and even moderate mass transfer can result in a significant spin up of the mass gainer (Langer 2004). There are many examples of rapid rotation in the gainer in Algol binary systems (van den Heuvel 1970; Etzel \& Olson 1993; Šimon 1999), and there is growing evidence that at least some fraction of the Be stars were spun up this way (Gies 2000, 2001; Maintz et al. 2004). Most of the massive X-ray binaries are found in the group of Be X-ray binaries that contain a Be star and neutron star companion (Coe 2000), and models of binary evolution suggest that the Be stars were spun up by mass transfer prior to the supernova event that formed the neutron star (van den Heuvel \& Rappaport 1987). One of best examples of this spin up process is found in the classical Be star, $\phi$ Persei $^{2}$. Gies et al. (1998) used UV spectroscopy from the Hubble Space Telescope to detect the faint mass donor, which is a hot subdwarf star, the stripped-down remains of the initially more massive component. The Be star was probably spun up by an earlier stage of mass transfer to attain the observed projected rotational velocity of $V \sin i=450$ $\mathrm{km} \mathrm{s}^{-1}$ (corresponding to $\approx 97 \%$ of the critical velocity). We do not know the incidence of binary spin up among massive stars because the companions in systems like $\phi$ Per are extremely difficult to detect.

Star clusters offer us the means to begin to sort out these various processes affecting rotation by showing us examples of stellar populations at various welldefined ages (established by the main sequence turn-off point). The main difficulty up to now has been the lack of significant numbers of clusters with large samples of $v \sin i$ measurements. The situation has changed with the advent of multiple-fiber spectroscopy which allows observers to obtain perhaps 100 stellar spectra in a single exposure of a cluster. We can anticipate an avalanche of new measurements in the near future, and below we describe some early results from a survey we have made.

\section{Preliminary Survey Results}

We are currently assembling measurements of $v \sin i$ for some 496 stars in 19 open clusters, which span an age range of 3 to $60 \mathrm{Myr}$. The spectra of the northern sky clusters were obtained with the WIYN 3.5-m telescope at Kitt Peak National Observatory while the southern clusters were observed with the Cerro Tololo Interamerican Observatory 4-m telescope. Both telescopes are equipped with Hydra multiple-object spectrographs (Barden \& Armandroff 1995) that use micropositioners and fiber optics to obtain spectra of many targets simultaneously. We obtained moderate dispersion spectra (resolving power of $R=5100$ and 3200 for WIYN and CTIO, respectively) that cover the blue spectral region. Our main goal was to record the absorption line profiles of $\mathrm{He} \mathrm{I} \lambda \lambda 4026,4387,4471$ that are relatively strong throughout the B-type spectral class. We generally observed each cluster field on at least two nights, so that we could make a preliminary

\footnotetext{
${ }^{2}$ See a painting at http://imgsrc.stsci.edu/op/pubinfo/PR/97/39/content/phiperp.jpg
} 
identification of the radial velocity variable stars (assumed to be spectroscopic binaries).

We fit the He I lines with model profiles based on the stellar atmosphere code TLUSTY and the radiative transfer code SYNSPEC to derive specific intensity profiles (Hubeny \& Lanz 1995). We constructed a grid of intensity profiles based on the parameters $\mu$, the cosine of the angle from the surface normal to the line of sight, $T_{\text {eff }}$, the local effective temperature, and $\log g$, the logarithm of the surface gravity. We then used used a synthesis code that integrates the line profile contributions over the visible hemisphere of the star. The code assumes Roche geometry to calculate the shape of the rotating star, and the integration weights each surface element using the appropriate degree of limb and gravity darkening. These are essentially "Collins models" (Collins 2004; Howarth 2004) but we made the simplifying assumption that the inclination of the spin axis is always $i=90^{\circ}$. Finally, the profiles were convolved with the instrumental broadening for direct comparison with the observed profiles. We found solutions for $v \sin i$ that minimized the difference between the observed and calculated profiles, and then formed an average based on all the available lines. The errors in $v \sin i$ are typically $\approx 5 \%$. We observed some 15 stars in common with the sample of Slettebak $(1968,1985)$ for the clusters NGC 869 and 884 (h and $\chi$ Per), and we find that our results are generally in good agreement with his except for a small offset:

$$
(V \sin i)[\text { Slettebak }]=0.999(V \sin i)[\text { Gies \& Huang }]+34 \mathrm{~km} \mathrm{~s}^{-1} .
$$

Our sample of open clusters is listed in Table 1 in order of increasing age (as given in the WEBDA database). We list the percentage of detected binary stars (double-lined or velocity variable targets), the total number of stars measured, the mean projected rotational velocity both for the whole sample and for those stars of subtype B3 and earlier, and the percentage of the sample with $V \sin i>300 \mathrm{~km} \mathrm{~s}^{-1}$ (the very fast rotators). The sample mainly consists of stars with MK spectral subtypes O9.5 - B8 and luminosity classes III - V. Figure 1 illustrates mean $v \sin i$ as function of spectral subtype with the total number of stars in each subtype given below (which shows that our sample is biased towards earlier subtypes). The distribution is basically flat over this range (similar to the classical diagram presented by Slettebak 1970) and does not indicate a trend towards faster rotation in the later subtypes (as found by Abt et al. 2002 in field stars and by Brown \& Verschueren 1997 in Sco OB2).

We plot in Figure 2 a histogram of the distribution of our observed $v \sin i$ for stars with subtypes between B0 and B7 (solid line). We also constructed a similar histogram for field B-type stars using the data from Abt et al. (2002) for stars with subtypes between B0 and B7 and luminosity classes between III and $\mathrm{V}$ (dashed line). It appears that the field stars are generally slower rotators than the cluster stars. The mean projected rotational velocities are $113 \pm 4$ and $160 \pm 4$ $\mathrm{km} \mathrm{s}^{-1}$ for the field and cluster stars, respectively (where the errors are the standard deviation of the mean). This difference between the field and cluster averages also appears in comparisons to other samples of field stars (Balona 1975; Wolff, Edwards, \& Preston 1982; Levato \& Grosso 2004). If we adopt the idea that most field stars represent the products of dispersed clusters, then perhaps the field stars represent a somewhat older population in which spin down processes have had more time to act. 
Table 1. Mean Projected Rotational Velocities in Open Clusters

\begin{tabular}{lcccccc}
\hline & $\begin{array}{c}\text { log Age } \\
\text { (Myr) }\end{array}$ & $\begin{array}{c}\text { Binary } \\
(\%)\end{array}$ & $\begin{array}{c}v \sin i \\
(\mathrm{All}) \\
\left(\mathrm{km} \mathrm{s}^{-1}\right)\end{array}$ & $\begin{array}{c}v \sin i \\
(\leq \mathrm{B} 3) \\
\left(\mathrm{km} \mathrm{s}^{-1}\right)\end{array}$ & $\begin{array}{c}V \sin i> \\
300 \mathrm{~km} \mathrm{~s}^{-1} \\
(\%)\end{array}$ \\
\hline Trumpler 16 & 6.43 & $\ldots$ & 36 & 155 & 167 & 8 \\
IC 1805 & 6.67 & 30 & 30 & 155 & 160 & 0 \\
Trumpler 14 & 6.67 & $\ldots$ & 5 & 128 & 189 & 0 \\
NGC 6193 & 6.72 & 20 & 20 & 174 & 159 & 15 \\
IC 2944 & 6.73 & 16 & 38 & 159 & 155 & 5 \\
NGC 2362 & 6.77 & 7 & 28 & 181 & 152 & 4 \\
NGC 2244 & 6.80 & 34 & 41 & 172 & 163 & 7 \\
NGC 2384 & 6.90 & 13 & 15 & 135 & 120 & 0 \\
NGC 1502 & 7.03 & 22 & 18 & 178 & 190 & 11 \\
NGC 3293 & 7.03 & 9 & 23 & 203 & 194 & 26 \\
Berkeley 86 & 7.05 & 6 & 17 & 200 & 187 & 18 \\
NGC 869 & 7.10 & 35 & 55 & 132 & 130 & 5 \\
NGC 457 & 7.15 & 42 & 19 & 160 & 182 & 11 \\
NGC 884 & 7.15 & 30 & 57 & 159 & 153 & 7 \\
NGC 2467 & 7.24 & 14 & 14 & 165 & 176 & 7 \\
NGC 4755 & 7.26 & 3 & 33 & 160 & 175 & 6 \\
IC 2395 & 7.35 & 12 & 16 & 137 & 127 & 0 \\
NGC 7160 & 7.37 & 25 & 16 & 188 & 162 & 6 \\
NGC 2422 & 7.78 & 13 & 15 & 150 & 150 & 0 \\
\hline \hline
\end{tabular}

Figure 1. Mean $v \sin i$ as a function of spectral subtype. The number of stars in each subtype bin is given at the bottom.

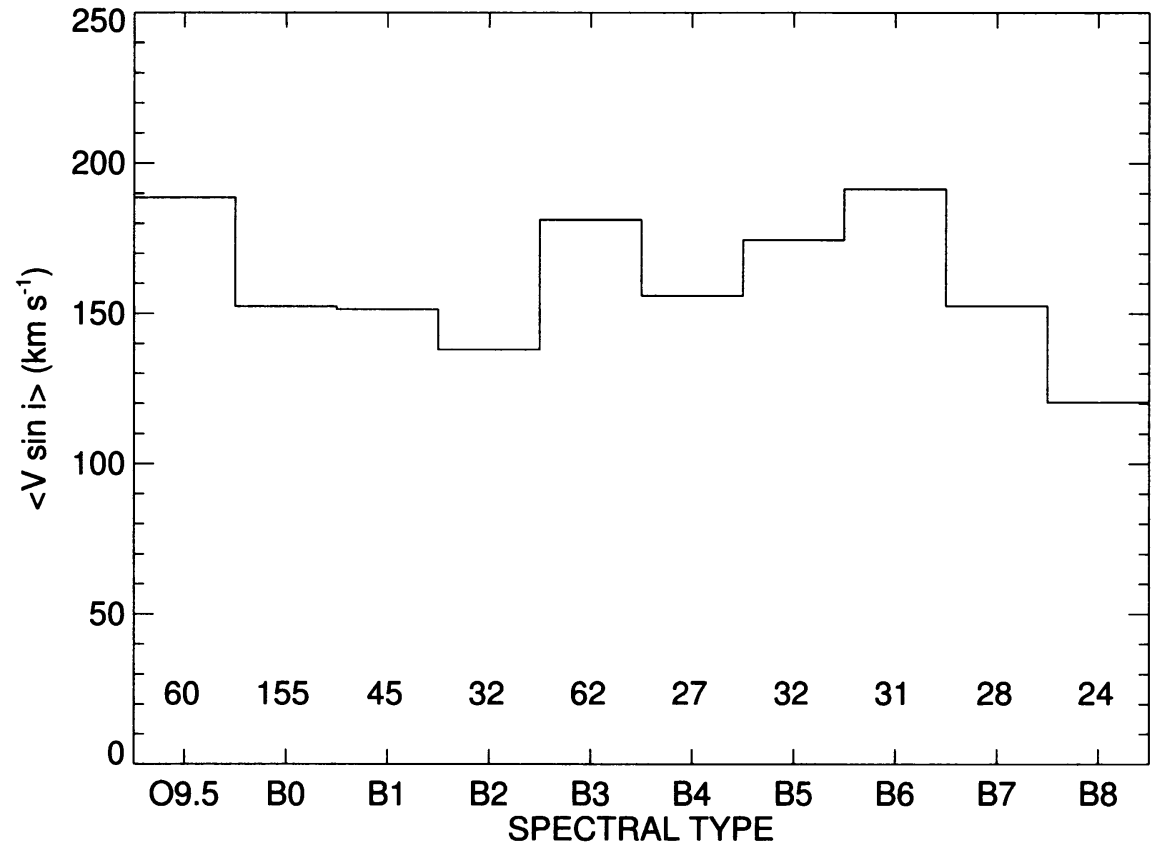


Figure 2. Histograms of $v \sin i$ for field stars from Abt et al. (2002) (dashed line) and for cluster stars (solid line).

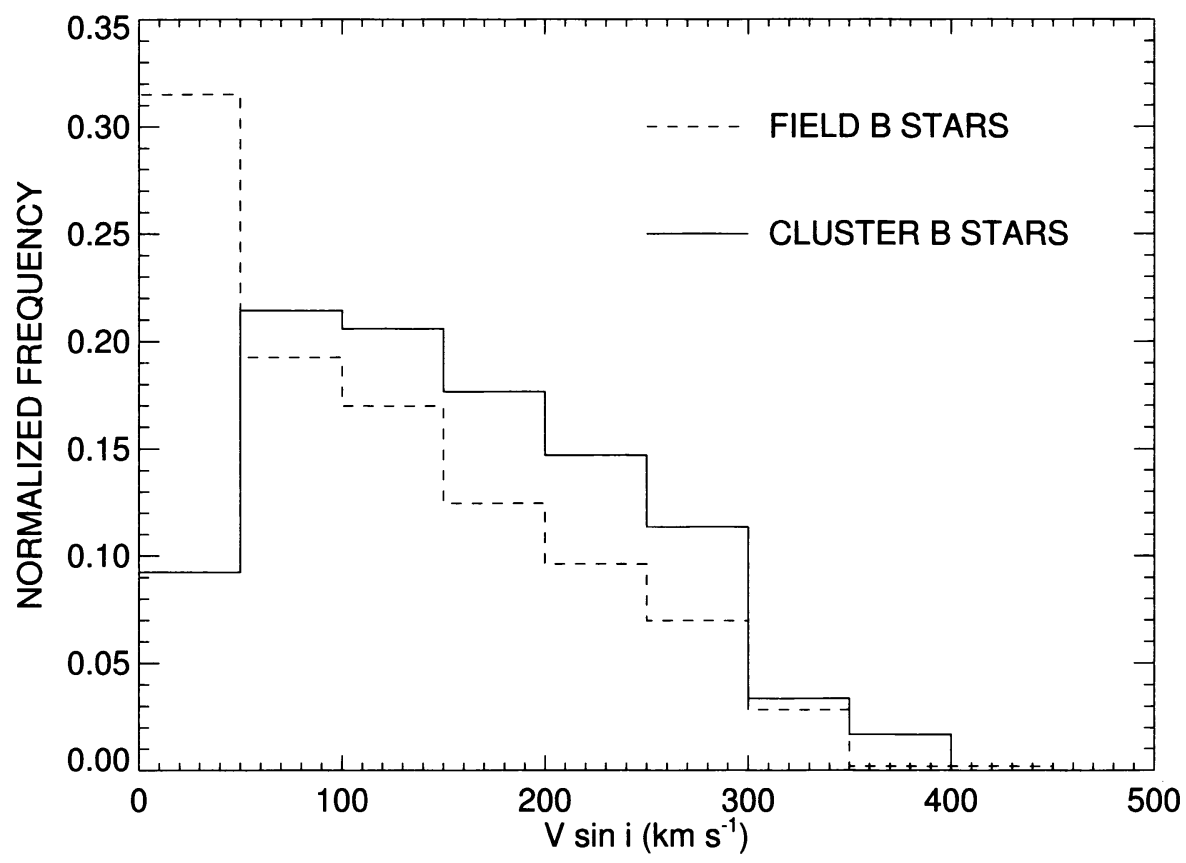

We confirm the trend found in many other investigations that binaries are slower rotators. The mean $v \sin i$ values are $168 \pm 4$ and $125 \pm 8 \mathrm{~km} \mathrm{~s}^{-1}$ for the constant and variable velocity stars, respectively. However, we find that there is no obvious anti-correlation between the incidence of detected binaries and mean $v \sin i$ in our sample. We suspect that our rudimentary method of estimating the binary population is probably insufficient to assess real differences in the binary statistics between clusters.

It is difficult to make a direct comparison of the predictions for evolutionary spin down with the cluster data because the spin down rates are related to both stellar mass and initial rotational speed. We have made a simplified analysis by restricting the sample to stars of subtype B3 and earlier where we expect the spin down rates to be largest. These cluster mean $v \sin i$ values are plotted in Figure 3 for clusters with log Age $(\mathrm{Myr})<7.5$ and with more than 7 measurements. The mean spectral subtype in this limited sample corresponds approximately to a star of mass $12 M_{\odot}$, so we used a model for this mass from Meynet \& Maeder (2000) to estimate the predicted spin down between ZAMS and TAMS. We assumed that all clusters begin with a distribution of stellar rotational velocities given by our histogram in Figure 2. Then, we used the Meynet \& Maeder (2000) model to calculate how each $50 \mathrm{~km} \mathrm{~s}^{-1}$ bin is transformed to a lower velocity at each time step in order to determine a new mean $v \sin i$ for that time step. This predicted decline is shown as the solid line in Figure 3 . Note that this basic calculation ignores the fact that the global $v \sin i$ distribution is actually based upon stars with a mixture of ages (including older, spun down 
Figure 3. Mean $v \sin i$ for stars earlier than or equal to spectral subtype B3 in young clusters plotted against logarithm of cluster age. The solid line shows the prediction from Meynet \& Maeder (2000) for an ensemble of $12 M_{\odot}$ stars normalized to the observed means for the youngest clusters.

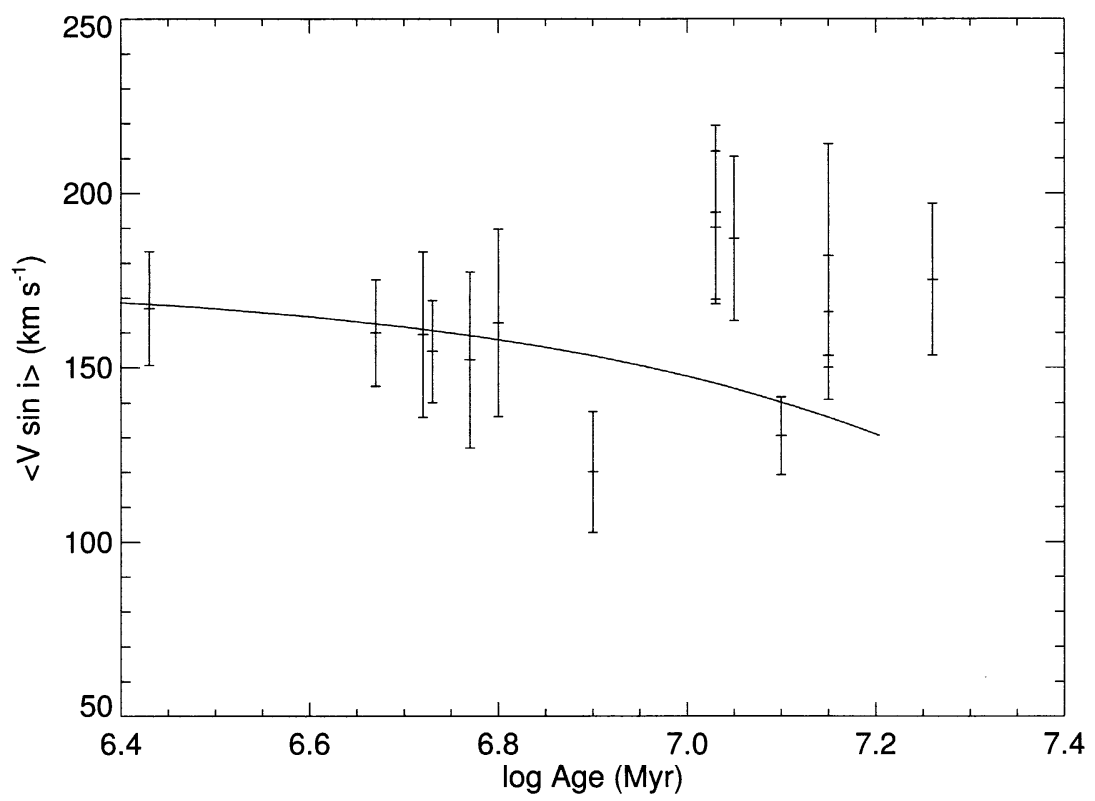

stars), and furthermore we have underestimated the actual velocity distribution by neglecting projection effects (i.e., by using a distribution of $v \sin i$ instead of $V)$. We attempted to correct for these deficiencies by applying a normalization factor of 1.1 to the predicted mean $v \sin i$ curve in order to better match the observed means in the youngest clusters. The predicted slow decline in $v \sin i$ with time is consistent with much of the cluster data, but there appears to be a surplus of clusters with rapid rotators in the age range $10-20$ Myr (see also the final column in Table 1). It is certainly possible that these deviations result from cluster-to-cluster differences in binary population, initial spin rates, and rotational dependence on mass, but taken at face value, these results suggest that some spin up processes become important in this time span.

Our record holder for fastest mean rotational velocity is NGC 3293, the oldest subgroup of the Car OB1 association ${ }^{3}$ (age of $11 \mathrm{Myr}$ ). This cluster contains several B supergiants and an $M$ supergiant. We found that 7 of the 23 stars we observed have $V \sin i>300 \mathrm{~km} \mathrm{~s}^{-1}$, and one additional fast rotator was found by Balona (1975). The positions of these stars in the HRD are shown in a color-magnitude diagram in Figure 4. Our targets were selected from the brightest stars, and observations of fainter stars by Royer et al. (2004) indicate that the rapid rotators are more prevalent among the brighter, more massive stars. Only one of the fast rotators in Figure 4 is found near the TAMS where

\footnotetext{
${ }^{3}$ See a color image at http://www.ast.cam.ac.uk/AA0/images/captions/aat010.html
} 
Figure 4. Color-magnitude diagram for the upper end of the main sequence of NGC 3293 (from photometry by Turner et al. 1980). The crosses enclosed in diamonds indicate the stars observed with $V \sin i \geq 300 \mathrm{~km} \mathrm{~s}^{-1}$.

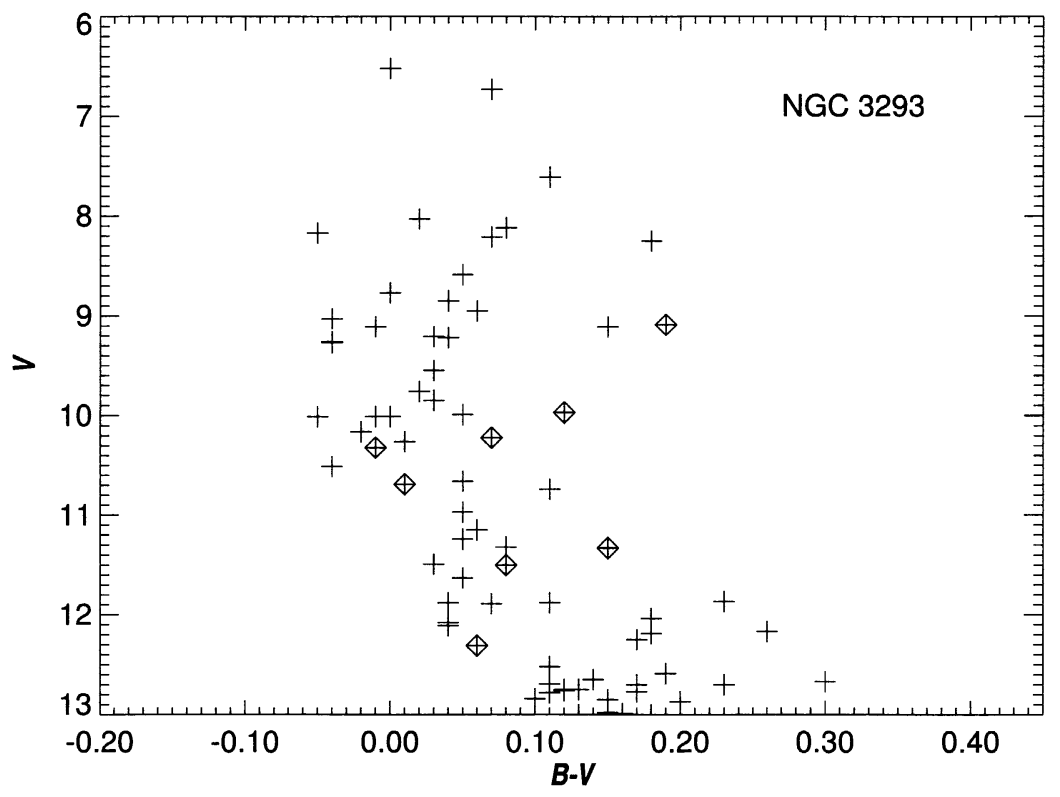

single star models indicate spin up may occur. This suggests that either an unusual number of stars were born close to the critical rotation rate in this cluster or that the cluster has evolved to the point where a number of close binary systems have undergone mass transfer leading to spin up in the mass gainer. It is interesting to note that we detected few binaries in this cluster perhaps because some of the original binaries are now post-Roche lobe overflow objects with very small orbital semi-amplitudes.

\section{Summary}

There are number of programs underway at the moment that will soon provide us with a large sample of projected rotational velocities for massive stars in open clusters (Corral, Herrero, \& Villamariz 2004; North et al. 2004; Royer et al. 2004). These studies should provide key insights about the relative importance of evolutionary changes, binaries, and magnetic fields in shaping the distributions of stellar rotational velocity. Preliminary results from our own survey offer some evidence that supports the predicted spin down in B stars during $\mathrm{H}$-core burning. In particular, the slower rotational velocities found in field versus cluster stars suggest that the somewhat older field population has experienced more prolonged spin down effects. Massive binaries clearly have lower $v \sin i$ values than single stars, but are they born this way or spun down by tidal action? Future studies of spectroscopic binaries in newborn clusters will help answer this question. Our results offer tentative support for the emergence of 
rapid rotators in some clusters in the age range $10-20 \mathrm{Myr}$. Some of these clusters may contain stars spun up by core contraction near the TAMS, but the rapid rotators might also be the result of binary mass transfer in systems that have recently experienced a first stage of Roche lobe overflow. An important test of these explanations will be a careful observational search for evidence of mass donors orbiting the rapid rotators (Maintz et al. 2003).

Acknowledgments. We thank the staffs of KPNO and CTIO for their help in obtaining the Hydra spectra. This material is based on work supported by the National Science Foundation under Grant No. AST-0205297. Support for this work was also provided by NASA through grant numbers GO-8308 and GO-9449 from the Space Telescope Science Institute, which is operated by the Association of Universities for Research in Astronomy, Inc., under NASA contract NAS526555. Institutional support has been provided from the GSU College of Arts and Sciences and from the Research Program Enhancement fund of the Board of Regents of the University System of Georgia, administered through the GSU Office of the Vice President for Research. We gratefully acknowledge all this support.

\section{References}

Abt, H. A. 1970, in Stellar Rotation, ed. A. Slettebak (Dordrecht: Reidel), 193

Abt, H. A., Levato, H., \& Grosso, M. 2002, ApJ 573, 359

Abt, H. A., \& Sanders, W. L. 1973, ApJ 186, 177

Abt, H. A., \& Willmarth, D. W. 1999, ApJ 521, 682

Balona, L. A. 1975, MNRAS 173, 449

Barden, S. C., \& Armandroff, T. 1995, in Proc. SPIE Vol. 2476, Fiber Optics in Astronomical Applications, ed. S. C. Barden (Bellingham, WA: SPIE), 56

Brown, A. G. A., \& Verschueren, W. 1997, A\&A 319, 811

Coe, M. J. 2000, in ASP Conf. Ser. 214, The Be Phenomenon in Early-type Stars, IAU Colloquium 175, ed. M. A. Smith, H. F. Henrichs, \& J. Fabregat (San Francisco: ASP), 656

Collins, A. G. W., II, 2004, in ASP Conf. Ser., Stellar Rotation, Proc. IAU Symp. No. 215, ed. A. Maeder \& P. Eenens (San Francisco: ASP), this volume

Corral, L., Herrero, A., \& Villamariz, R. 2004, in ASP Conf. Ser., Stellar Rotation, Proc. IAU Symp. No. 215, ed. A. Maeder \& P. Eenens (San Francisco: ASP), this volume

Etzel, P. B., \& Olson, E. C. 1993, AJ 106, 1200

Fabregat, J., \& Torrejón, J. M. 2000, A\&A 357, 451

Gies, D. R. 2000, in ASP Conf. Ser. 214, The Be Phenomenon in Early-type Stars, IAU Colloquium 175, ed. M. A. Smith, H. F. Henrichs, \& J. Fabregat (San Francisco: ASP), 668

Gies, D. R. 2001, in ASSL Vol. 264, The Influence of Binaries on Stellar Population Studies, ed. D. Vanbeveren (Dordrecht: Kluwer), 95

Gies, D. R., Bagnuolo, W. G., Jr., Ferrara, E. C., Kaye, A. B., Thaller, M. L., Penny, L. R., \& Peters, G. J. 1998, ApJ 493, 440

Heger, A., \& Langer, N. 2000, ApJ 544, 1016

Howarth, I. D. 2004, in ASP Conf. Ser., Stellar Rotation, Proc. IAU Symp. No. 215, ed. A. Maeder \& P. Eenens (San Francisco: ASP), this volume 
Hubeny, I., \& Lanz, T. 1995, ApJ 439, 875

Langer, N. 2004, in ASP Conf. Ser., Stellar Rotation, Proc. IAU Symp. No. 215, ed. A. Maeder \& P. Eenens (San Francisco: ASP), this volume

Levato, H., \& Grosso, M. 2004, in ASP Conf. Ser., Stellar Rotation, Proc. IAU Symp. No. 215, ed. A. Maeder \& P. Eenens (San Francisco: ASP), this volume

Levato, H., Malaroda, S., \& Morrell, N. I. 1991, in ASP Conf. Ser. 13, The Formation and Evolution of Star Clusters, ed. K. Janes (San Francisco: ASP), 411

Maeder, A., Grebel, E. K., \& Mermilliod, J.-C. 1999, A\&A 346, 459

Maintz, M., Rivinius, Th., Štefl, S., \& Stahl, O. 2004, in ASP Conf. Ser., Stellar Rotation, Proc. IAU Symp. No. 215, ed. A. Maeder \& P. Eenens (San Francisco: ASP), this volume

Mathieu, R. D. 2004, in ASP Conf. Ser., Stellar Rotation, Proc. IAU Symp. No. 215, ed. A. Maeder \& P. Eenens (San Francisco: ASP), this volume

Mathys, G. 2004, in ASP Conf. Ser., Stellar Rotation, Proc. IAU Symp. No. 215, ed. A. Maeder \& P. Eenens (San Francisco: ASP), this volume

Mermilliod J.-C. 1982, A\&A 109, 48

Mermilliod J.-C. 1995, in ASP Conf. Ser. 90, The Origins, Evolution, and Destinies of Binary Stars in Clusters, ed. E. F. Milone \& J.-C. Mermilliod (San Francisco: ASP), 475

Meynet, G., \& Maeder, A. 2000, A\&A 361, 101

Morrell, N., \& Levato, H. 1991, ApJS 75, 965

Neiner, C. 2002, Ph.D. thesis, University of Amsterdam

North, P., et al. 2004, in ASP Conf. Ser., Stellar Rotation, Proc. IAU Symp. No. 215, ed. A. Maeder \& P. Eenens (San Francisco: ASP), this volume

Rieutord, M. 2004, in ASP Conf. Ser., Stellar Rotation, Proc. IAU Symp. No. 215, ed. A. Maeder \& P. Eenens (San Francisco: ASP), this volume

Royer, F., Melo, C., Mermilliod, J.-C., North, P., do Nascimento, J. D., Jr., de Medeiros, J. R., Grebel, E., \& Maeder, A. 2004, in ASP Conf. Ser., Stellar Rotation, Proc. IAU Symp. No. 215, ed. A. Maeder \& P. Eenens (San Francisco: ASP), this volume

Šimon, V. 1999, A\&AS, 134, 1

Slettebak, A. 1968, ApJ 154, 933

Slettebak, A. 1970, in Stellar Rotation, ed. A. Slettebak (Dordrecht: Reidel), 3

Slettebak, A. 1985, ApJS 59, 769

Stauffer, J. R. 2004, in ASP Conf. Ser., Stellar Rotation, Proc. IAU Symp. No. 215, ed. A. Maeder \& P. Eenens (San Francisco: ASP), this volume

Turner, D. G., Grieve, G. R., Herbst, W., \& Harris, W. E. 1980, AJ 85, 1193

van den Heuvel, E. P. J. 1970, in Stellar Rotation, ed. A. Slettebak (Dordrecht: Reidel), 178

van den Heuvel, E. P. J., \& Rappaport, S. 1987 in Physics of Be Stars, ed. A. Slettebak \& T. P. Snow (Cambridge: Cambridge University Press), 291

Wolff, S. C., Edwards, S., \& Preston, G. W. 1982, ApJ 252, 322

Zorec, J. 2004, in ASP Conf. Ser., Stellar Rotation, Proc. IAU Symp. No. 215, ed. A. Maeder \& P. Eenens (San Francisco: ASP), this volume 